\title{
Direct interaction of FliX and FlbD is required for their regulatory activity in Caulobacter crescentus
}

Zhaohui $\mathrm{Xu}^{1,2^{*}}$, Rachel J Dutton ${ }^{1,3}$ and James W Gober ${ }^{1}$

\begin{abstract}
Background: The temporal and spatial expression of late flagellar genes in Caulobacter crescentus is activated by the transcription factor FlbD and its partner trans-acting factor FliX. The physical interaction of these two proteins represents an alternative mechanism for regulating the activity of $\sigma^{54}$ transcription factors. This study is to characterize the interaction of the two proteins and the consequences of the interaction on their regulatory activity.

Results: FliX and FlbD form stable complexes, which can stand the interference of $2.65 \mathrm{M} \mathrm{NaCl}$. The stability of FliX and FlbD was affected by the co-existence of each other. Five FliX mutants (R71A, L85K, $\triangle 117-118, T 130 \mathrm{~L}$, and L136K) were created by site-directed mutagenesis in conserved regions of the protein. All mutants were successfully expressed in both wild-type and $\Delta$ fliX Caulobacter strains. All but FliX $\mathrm{L}_{\mathrm{85K} K}$ could rescue the motility and cell division defects of a $\Delta$ fliX mutant strain. The ability of FliX to regulate the transcription of class $I I$ and class III/IV flagellar promoters was fully diminished due to the L85K mutation. Co-immunoprecipitation experiment revealed that FliX $\mathrm{L}_{\text {85K }}$ was unable to physically interact with FlbD.

Conclusions: FliX interacts with FlbD and thereby directly regulates the activity of FlbD in response to flagellar assembly. Mutations in highly conserved regions of FliX could severely affect the recognition between FliX and $\mathrm{FlbD}$ and hence interrupt the normal progression of flagellar synthesis and other developmental events in Caulobacter.
\end{abstract}

\section{Background}

Caulobacter crescentus undergoes a series of programmed differentiation events within each cell cycle and generates two dissimilar progeny cells, a motile swarmer cell possessing a single polar flagellum and a sessile stalked cell. A hallmark of this asymmetric cell division event is the temporal expression and asymmetric targeting of regulatory proteins as well as proteins comprising cellular structures such as the flagellum [1-5]. Over fifty genes are required for flagellar biogenesis in C. crescentus, and their temporal and spatial expression is regulated by both cell cycle events and the progression of flagellum assembly. Epistasis experiments have revealed that flagellar gene expression is subject to a regulatory hierarchy that reflects the assembly sequence of major flagellum sub-structures [6-15].

\footnotetext{
* Correspondence: zxu@bgsu.edu

'Department of Chemistry and Biochemistry and Molecular Biology Institute, University of California, Los Angeles, CA 90095-1569, USA

Full list of author information is available at the end of the article
}

The expression of the early flagellar genes (class II) encoding components of basal body switch, MS-ring, and flagellum-specific type-three secretion system (TTSS) is regulated by the timed synthesis and phosphorylation of the transcription factor CtrA [16-18]. The polar assembly of the MS-ring/switch/TTSS complex is required, in turn, for the transcription of genes (class III) encoding structures such as the rod, outer membrane rings, and the hook $[8,10,13,14]$. Finally, the complete construction of these class III-encoded structures are required to derepress the translation of flagellin mRNA (class IV), leading to the assembly of flagellar filament structure [19-22]. Thus, during C. crescentus flagellar biogenesis two different regulatory checkpoints link structural assembly to flagellar gene expression.

The transcription of class III and IV flagellar genes requires $\sigma^{54}$-containing RNA polymerase and the DNA binding protein, integration host factor (IHF) [23-28]. Transcription of these flagellar genes is under cell cycle control and, late in the cell cycle, is restricted to the
C Biomed Central

C 2011 Xu et al; licensee BioMed Central Ltd. This is an Open Access article distributed under the terms of the Creative Commons Attribution License (http://creativecommons.org/licenses/by/2.0), which permits unrestricted use, distribution, and reproduction in any medium, provided the original work is properly cited. 
swarmer cell compartment of the predivisional cell. This temporal and spatial transcription is regulated by FlbD, a $\sigma^{54}$ transcription factor [29-34]. The conserved receiver domains of this class of proteins are usually phosphorylated by a cognate sensor histidine kinase, which in turn stimulates oligomerization and DNA-binding of these proteins at enhancer sequences. Rather than phosphrylation, FlbD activity is regulated by FliX, a conserved trans-acting factor that is present in polarly flagellated $\alpha$-proteobacteria and has no demonstrated histidine kinase activity [35-38]. Caulobacter strains bearing mutations in $f l i X$ are non-motile and do not transcribe class III and IV flagellar genes. Gain-of-function mutations in FlbD can by-pass the transcriptional requirement for FliX, suggesting that FliX is a trans-acting factor rather than a structural component of the flagellum [36]. Additionally, FliX enhances FlbD-activated transcription in vitro by stimulating purified FlbD to form higher-order oligomers [35]. Interestingly, overexpression of FliX suppresses FlbD-activated transcription in vivo, and a mutant allele of $f l i X, f l i X 1$, has been isolated that can by-pass the early flagellar assembly requirement for class III and IV transcription [38]. These observations suggest that upon the complete assembly of an early class II flagellar basal body structure, FliX switches from a negative to a positive regulator of FlbD.

The physical interaction of FliX and FlbD represents a novel mechanism for regulating the activity of a $\sigma^{54}$ transcription factor [35]. Here, we describe a genetic and biochemical analysis dissecting the role of FliX in regulating FlbD activities. We present evidence that FliX and FlbD are in stable complexes under physiological conditions. Furthermore, we show that highly-conserved regions of FliX are critical for its productive interaction with FlbD and for proper regulation of flagellar gene expression in response to the progression of flagellar assembly.

\section{Methods}

\section{Bacterial strains and plasmids}

Bacterial strains and plasmids involved in this work are summarized in Table 1. Caulobacter crescentus strains were grown in peptone-yeast extract (PYE) [39] at $31^{\circ} \mathrm{C}$. Antibiotics were supplemented when necessary to a final concentration of $2.5 \mu \mathrm{g} / \mathrm{ml}$ of chloramphenicol, 2 $\mu \mathrm{g} / \mathrm{ml}$ of tetracycline, or $20 \mu \mathrm{g} / \mathrm{ml}$ of nalidixic acid. PYE motility plates contained $0.3 \%(\mathrm{w} / \mathrm{v})$ agar. E. coli strains were grown at $37^{\circ} \mathrm{C}$ in Luria-Bertani broth supplemented with one or more of the following antibiotics:

Table 1 Bacterial strains and plasmids used in this study

\begin{tabular}{|c|c|c|}
\hline $\begin{array}{l}\text { Strains or } \\
\text { plasmids }\end{array}$ & Genotypes or descriptions & Sources \\
\hline \multicolumn{3}{|l|}{ C. crescentus } \\
\hline LS107 & syn-1000, bla-6, amps derivative of NA1000 & Stephens et al. [45] \\
\hline$J G 1172$ & syn-1000 bla-6 $\Delta$ flix & Muir et al. [38] \\
\hline SC1032 & flbD198:: $\operatorname{Tn} 5$ & Ohta et al. [41] \\
\hline \multicolumn{3}{|l|}{ E. coli } \\
\hline S17-1 & Rp4-2, Tc::Mu, Km::Tn7 & Simon et al. [46] \\
\hline $\mathrm{BL} 21(\mathrm{DE} 3)$ & 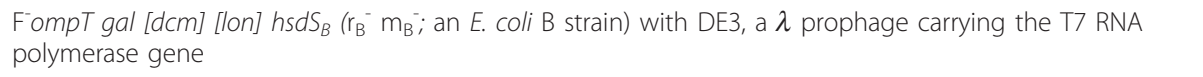 & Novagen \\
\hline \multicolumn{3}{|l|}{ Plasmids } \\
\hline $\mathrm{p} \times 21 \mathrm{~b}$ & derivative of pET-21b carrying histidine-tagged FliX under the control of T7 promoter, Ap ${ }^{r}$ & Muir \& Gober [36] \\
\hline pBBR1MCS & broad host range cloning vector, multicopy, $\mathrm{Cm}^{\mathrm{r}}$ & Kovach et al. [47] \\
\hline pZXfliX & $\begin{array}{l}\text { derivative of pBBR1MCS carrying PCR generated } 894 \text { bp BamHI-HindIII fragment containing fliX gene and } \\
\text { its promoter }\end{array}$ & This study \\
\hline $\mathrm{pZXX71A}$ & derivative of pZXfliX, codon $71 \mathrm{cgcc} \rightarrow \mathrm{gcc}$ & This study \\
\hline pZX85K & derivative of pZXfliX, codon 85 ctg $\rightarrow$ aag & This study \\
\hline $\mathrm{pZX} \triangle 117-8$ & derivative of pZXfliX, deletion of codon 117 and 118 & This study \\
\hline pZX130L & derivative of pZXfliX, codon $130 \mathrm{acc} \rightarrow \mathrm{ctg}$ & This study \\
\hline pZX136K & derivative of pZXfliX, codon 136 ctg $\rightarrow$ aag & This study \\
\hline pflix1 & $\begin{array}{l}\text { derivative of pBBR1-MCS expressing flix 1, an allele of flix carrying a point mutation at the sixteenth } \\
\text { codon (R16G), a frame shift at codon 141, and an extended carboxyl terminus of } 67 \text { amino acids }\end{array}$ & Muir et al. [38] \\
\hline pfliF/lacZ/290 & fliF-lacZ transcriptional reporter vector, $T c^{r}$ & Wingrove \& Gober [48] \\
\hline pfliK/lacZ/290 & flik-lacZ transcriptional reporter vector, $T c^{r}$ & Gober \& Shapiro [25] \\
\hline
\end{tabular}


chloramphenicol $(30 \mu \mathrm{g} / \mathrm{ml})$, tetracycline $(12.5 \mu \mathrm{g} / \mathrm{ml})$, or ampicillin $(50 \mu \mathrm{g} / \mathrm{ml})$. DNA manipulations were carried out according to standard procedures. Plasmids were introduced into $C$. crescentus by conjugation with E. coli $\mathrm{S} 17-1$.

\section{Identification of FliX-bound proteins with mass spectrometry}

About 1.64 g of CNBr-activated sepharose 4B beads (GE Healthcare, Piscataway, NJ, USA) were swelled and washed as recommended by the manufacture and incubated overnight with $36.6 \mathrm{mg}$ of histidine-tagged FliX (FliX-His) that was prepared as previously described [35]. After incubation at $4^{\circ} \mathrm{C}$ with end-over-end rotation, the bead complexes were alternately washed with acidic buffer $(0.1 \mathrm{M}$ acetate, $0.5 \mathrm{M} \mathrm{NaCl}, \mathrm{pH} 4.0)$ and alkaline buffer (90 mM Tris.Cl, $0.5 \mathrm{M} \mathrm{NaCl}, \mathrm{pH} 8.5$ ) for 3 cycles. Such prepared sepharose-FliX complexes were then conditioned by PBS buffer $(0.1 \mathrm{M}$ sodium phosphate, 0.15 $\mathrm{M} \mathrm{NaCl}, \mathrm{pH} \mathrm{7.2)}$ and stored at $4{ }^{\circ} \mathrm{C}$ for later use. Meanwhile, 5 liters of $C$. crescent LS107 culture was harvested by centrifugation, resuspended in $100 \mathrm{ml}$ of PBS buffer, lysed by French Press, and centrifuged at 26,690 $g$ for 1 h. The supernatant was mixed with the above sepharose-FliX complexes and incubated at $4^{\circ} \mathrm{C}$ for overnight with gentle rocking. Cell extract was then removed by centrifugation. The pellet containing the sepharose bead complexes was washed with $20 \mathrm{ml}$ of PBS buffer for three times and resuspended in $5 \mathrm{ml}$ of the same buffer. An aliquot of $100 \mu \mathrm{l}$ was removed and boiled with loading buffer for sodium dodecyl sulfate-polyacrylamide gel electrophoresis (SDS-PAGE) analysis. The gel was visualized with Coomassie staining. The apparent bands were excised, partially digested with trypsin, and were analyzed by electrospray ionization (ESI)-ion trap mass spectrometry at Stanford University http://mass-spec. stanford.edu/.

\section{Stability assays of FliX and FlbD}

Protein synthesis in cultures grown to mid-log phase was inhibited by addition of chloramphenicol to a final concentration of $3 \mathrm{mg} / \mathrm{ml}$. One milliliter of cell culture was taken at $0,15,30$, and 45 min after the addition of the antibiotic. Cell pellets were electrophoresed in $12 \%$ $(\mathrm{w} / \mathrm{v})$ polyacrylamide gels and were analyzed using antiFlbD or anti-FliX antibodies.

\section{Site-directed mutagenesis of fliX}

A fragment of 894 bp covering the coding sequence of fliX and its promoter region was amplified by PCR from C. crescentus chromosome and was inserted into pBBR1MCS to give raise to pZXfliX, which was then used as the template to create fliX mutants. For every mutant to be generated, two pairs of primers were designed to amplify the two fragments covering each side of the desired mutation site. The intended mutation sequence was overhung at the $5^{\prime}$ end of the downstream fragment. For the convenience of manipulation, BamHI recognition sequence was engineered at the $5^{\prime}$ end of the upstream fragment, and HindIII at the 3' end of the downstream fragment. The two fragments were then phosphorylated, treated with BamHI or HindIII, and inserted into pBBR1MCS to generate pZX series plasmids (Table 1). All mutants were confirmed by DNA sequencing.

\section{Protein expression analysis of FlbD and the FliX alleles}

Overnight cultures of $C$. crescentus were transferred to fresh PYE media at a ratio of 1 to $10(\mathrm{v} / \mathrm{v})$ and were allowed to grow at $31^{\circ} \mathrm{C}$ until mid-log phase. Culture biomass was measured as optical density at $600 \mathrm{~nm}$ $\left(\mathrm{OD}_{600}\right)$, normalized, and was subject to $14 \%(\mathrm{w} / \mathrm{v})$ SDSPAGE. After electrophoresis, protein profiles were transferred to nitrocellulose membranes and were detected using anti-FliX or anti-FlbD antibodies purified with affinity columns (AminoLink ${ }^{\circledR}$ Plus Immobilization Kit, Thermo Fisher Scientific Inc., Rockford, IL, USA).

\section{Measurement of the transcription of flagellar genes}

The pZX serial plasmids bearing various fliX mutants were introduced into the wild-type strain LS107 or the $\Delta f l i X$ stain JG1172 via conjugation, along with the reporter genes fliF-lacZ or fliK-lacZ. $\beta$-Galactosidase activity was measured as described previously [40].

\section{Co-immunoprecipitation (co-IP)}

Cells in middle log stage were harvested, normalized, and treated with $5 \mathrm{mg} / \mathrm{ml}$ lysozyme. The clear cell extract was incubated with Agarose-Protein A beads (Roche Applied Science, Indianapolis, IN, USA) to eliminate non-specific associated proteins. The pre-cleared cell lysate was then incubated overnight with AgaroseProtein A-anti-FlbD complexes prepared as instructed by the manufacturer. After extensive washing, the bead complexes were spun down, resuspended in SDS-PAGE sample buffer and were subjected to electrophoresis followed by immunoblotting with anti-FliX antibodies.

\section{Results}

FlbD forms stable in vivo complex with Flix

Previous experiments have shown that FliX and FlbD interact in a two-hybrid assay [37], FliX can be precipitated from cell extracts of Caulobater by anti-FlbD antibodies, and that FliX regulates FlbD-activated transcription in vitro [35]. In order to gain further understanding of the physical recognition between the two and to find out whether there are other proteins associated with FliX-FlbD complex, we performed an 
affinity pull-down experiment in which cell extracts of Caulobacter were treated with sepharose beads coated with histidine-tagged wild-type FliX. Cellular proteins that physically associated to FliX were then retrieved from the bead complexes and resolved by electrophoresis (Figure 1). Five major bands with corresponding molecular weights of approximately 70, 60, 48, 44, and 19 kilodaltons were observed. Mass spectrometry analysis revealed that these proteins were heat shock proteins DnaK and CH60 (GroEL), FlbD, elongation factor EF$\mathrm{Tu}$, and FliX-His, respectively. Proteins DnaK, CH60, and EF-Tu are among the most abundant cellular proteins found in bacteria, including those possessing no flagellum. It is unlikely that these proteins would interact with FliX in a specific manner. Furthermore, when washing the sepharose bead complexes with phosphate buffer containing $\mathrm{NaCl}$ ranging from 0.3 to $2.65 \mathrm{M}$, these three proteins were readily released to the washing buffer throughout the salt gradient, whereas no FlbD or FliX protein could be washed off even with the highest salt strength used. The co-occurrence of FliX and FlbD in the sepharose bead complexes demonstrates that FlbD indeed directly interacts with FliX inside of Caulobacter cells, and that the affinity between the two proteins is remarkably high. We did not observe any other

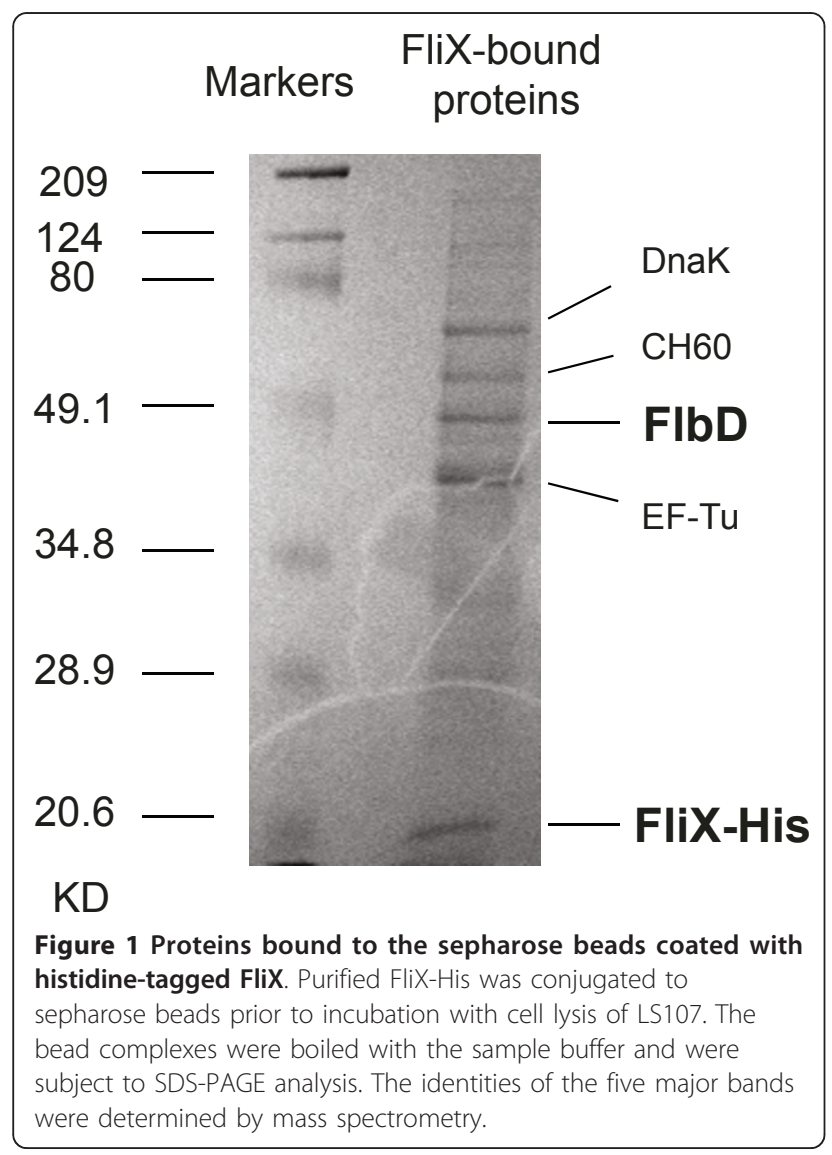

major specific component of the FlbD-FliX complex, although we cannot rule out the possibility that there might be transiently associated proteins, which are not detectable by the method described here.

\section{Interaction between FlbD and FliX is required for stabilizing each other in vivo}

The finding that FlbD and FliX form high affinity in vivo complexes motivated us to examine whether the two proteins depend on each other for existence. We assayed the half-life of each protein in a wild-type Caulobacter strain (LS107), a strain bearing a deletion in fliX (JG1172), and a strain having a Tn5 insertion in flbD (SC1032). Chloramphenicol was added to cell cultures at mid-log phase to inhibit protein synthesis, and the protein contents of FlbD and FliX were analyzed periodically. In strain LS107, both FlbD and FliX were stable; neither exhibited significant reduction in concentration following $45 \mathrm{~min}$ of exposure to chloramphenicol (Figure 2). In contrast, after $45 \mathrm{~min}$, less than $40 \%$ of FlbD remained in strain JG1172. Likewise, a similar decrease in FliX level was evident in SC1032 cells. These results indicate that FlbD has a reduction in stability in the absence of FliX, and vice versa.

\section{Site-directed mutagenesis of FliX}

To learn more about the interaction between FliX and FlbD, we performed site-directed mutagenesis with fliX and investigated the effects of mutations on FlbD activity. Both FlbD and FliX homologs are present in dozens of $\alpha$-proteobacteria species that possess polar flagella. Among the FliX homologs, the central (residues 53-95) and carboxyl-terminal (residues 116-142) regions are

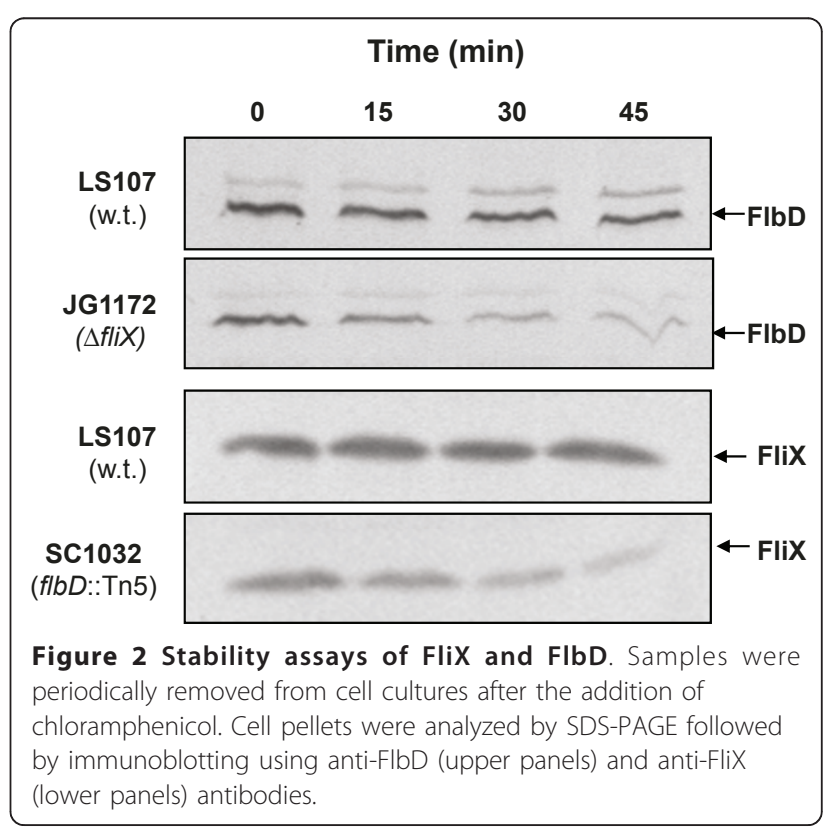


highly conserved (Figure 3). To determine the roles of these regions in FliX functionality, five conserved sites were selected as the target sites for mutation: R71, L85, D117-D118, T130, and L136 (Figure 3). In the region from amino acids 69 to 73, there are five consecutive charged residues. This pattern is less common in protein sequences and may be important for FliX activity; so we chose to replace the central residue $\mathrm{R} 71$ with alanine to disrupt this pattern. We also deleted residues D117 and D118 in order to abolish these potential phosphorylation sites. In addition, we noticed that the 130th residue of FliX is a threonine, which is different from the majority of its homologs where a leucine is found. We then replaced T130 with an L and hoped to create a "super" FliX, because a conserved residue in a given position is often the most suitable one. Finally, we replaced $\mathrm{L}$ with $\mathrm{K}$ at sites 85 and 136 with the intention to disrupt any potential secondary structures of the conserved regions. Plasmid bearing either the wild-type or a mutant fliX allele, along with the $f l i X$ promoter region, was introduced into LS107 (wild-type strain) and JG1172 ( $\Delta$ fliX strain) for further analyses.

Role of conserved FliX residues in protein expression We first examined the expression of the FliX alleles and FlbD. Cell extracts were subject to SDS-PAGE analysis followed by immunoblotting with anti-FliX and antiFlbD antibodies (Figure 4). Strain SC1032 (flbD::Tn5) [41] and a constitutively active $f l i X$ allele $(f l i X 1)$, which carries an extended carboxyl terminus [38], were also included as controls. As was previously reported [36], the flbD::Tn5 cells possessed markedly reduced levels of FliX (lane 1); similarly, $\Delta$ cells contained little FlbD (lane 10). These observations are also in support of the findings that FlbD and FliX interact with each other in vivo (Figure 1) and that the absence of either protein reduces the stability of the other (Figure 2). In both LS107 and JG1172 cells, FliX $\mathrm{R}_{11 \mathrm{~A}}$, FliX $\mathrm{T}_{\mathrm{T} 130 \mathrm{~L}}$, and FliX $\mathrm{L}_{\mathrm{L} 136 \mathrm{~K}}$ were present at levels comparable to wild-type FliX carried on a multi-copy plasmid (Figure 4, lanes 3 and 11). However, the concentrations of FliX $\mathrm{L}_{\mathrm{LSK}}$ and FliX $\mathrm{Cl17-118}$ in JG1172 cells were significantly reduced (greater than ten-fold) compared to other FliX mutants; the FlbD levels in these cells were also diminished (lane 13 and 14). Nevertheless, all mutants were successfully expressed in both wild-type and $\Delta$ fliX strains. We next examined the effects of these mutations on FlbD-mediated cellular processes.

\section{Role of conserved FliX residues in flagellar synthesis}

Cells expressing each $f l i X$ allele were tested for motility using soft agar plates, on which motile cells swim away from the point of inoculation, forming a visible halo. In LS107 cells, the over-expression of either wild-type or mutant alleles of $f l i X$ from a multi-copy plasmid resulted in reduced swarm sizes, indicating that motility was slightly impaired by the over-expression (Figure 5). In JG1172 cells, all fliX alleles but $f l i X_{\mathrm{L} 85 \mathrm{~K}}$ were able to restore motility to the $\Delta$ fliX host (Figure 5); mutant

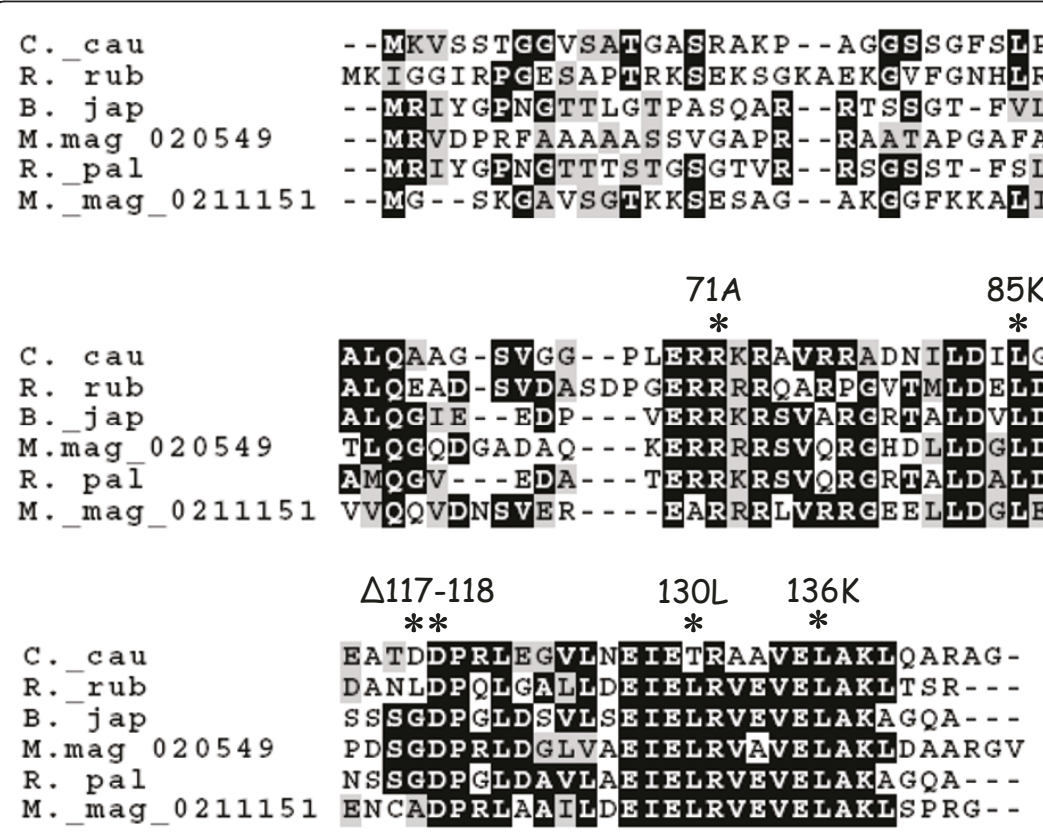

Figure 3 Site-directed mutagenesis of $\boldsymbol{C}$. crescentus FliX. Homologs of $C$. crescentus FliX are aligned with CLUSTAL W 1.81 and are shaded with BOXSHADE 3.3.1. Black, identical residues; grey, similar residues; asterisks, sites of mutation. C._ cau: C. crescentus, R. rub: Rhodospirillum rubrum, B. jap: Bradyrhizobium japonicum, M. mag: Magnetospirillum magnetotacticum, and R. _pal: Rhodopseudomonas palustris. 


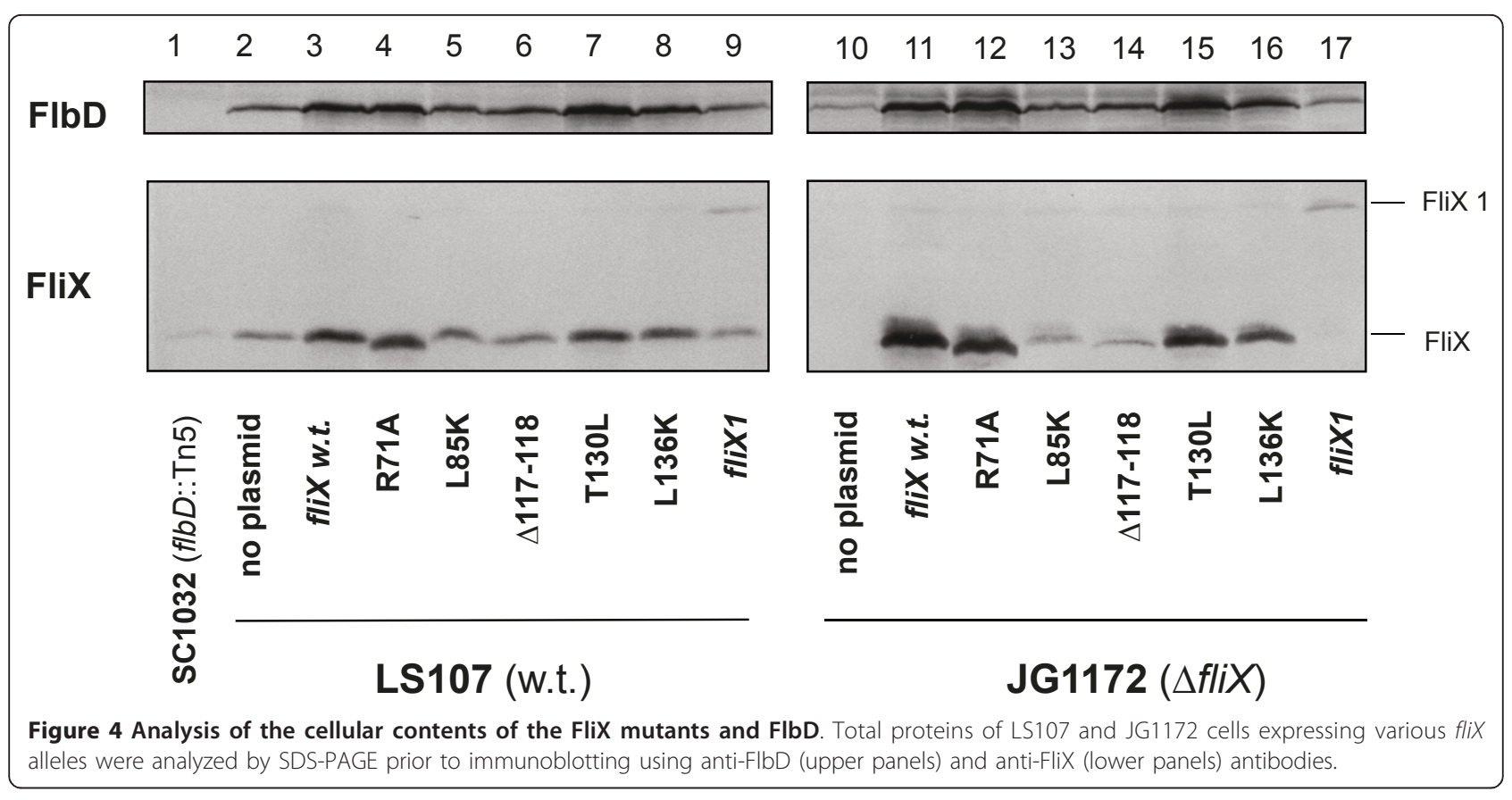

$f l i X_{\Delta 117-118}$ resulted in the smallest swarm size. Since fli $X_{\mathrm{L} 85 \mathrm{~K}}$ and $f l i X_{\Delta 117-118}$ were found at similar levels in JG1172 cells, it was intriguing to notice that the two mutants rendered distinctive physiological properties to their host cells.

Previous experiments indicate that FliX functions as a positive regulator of FlbD activity [38]. In order to find out whether $f l i X_{\mathrm{L} 85 \mathrm{~K}}$ and $f l i X_{\Delta 117-118}$ can effectively regulate FlbD-mediated transcription of flagellar genes, the two mutants were introduced into LS107 and JG1172 cells that also contained either a fliF- (class II) or a fliKlacZ (class III) transcriptional reporter fusion. When no fliX plasmid was involved, $\beta$-galactosidase activity generated from the fliF promoter was increased (Figure 6A)

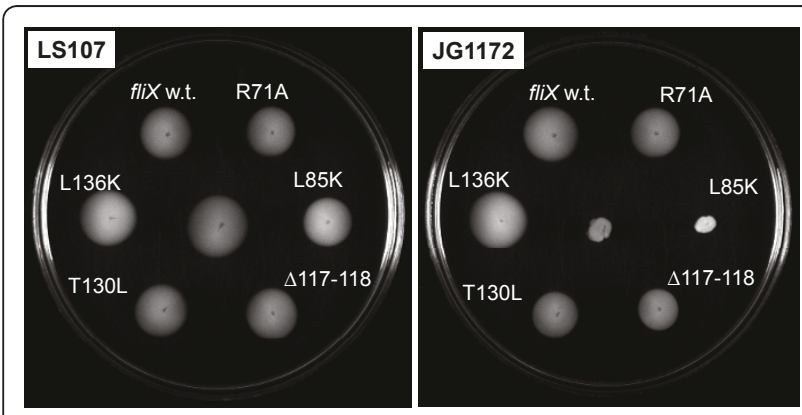

Figure $\mathbf{5}$ Motility of the cells harboring various fliX alleles. Cells were inoculated in motility agar and were incubated at $31^{\circ} \mathrm{C}$ for 3 days. Motile cells swarming away from the points of inoculation are visible as halos. Host strains containing no plasmid reside at the center of each plate. and from the fliK promoter (Figure 6B) was reduced in JG1172 cells compared to LS107 cells. This is in agreement with previous findings that FlbD represses the transcription of class II genes and activates the expression of class III genes [36]. In both LS107 and JG1172 backgrounds, transcriptional activity from either promoter in cells expressing $f l i X_{L 85 K}$ was equivalent to that obtained in cells carrying no plasmid (Figure 6), suggesting that this fliX allele was completely impaired in activating FlbD. In both wild-type and $\Delta f l i X$ cells, mutant FliX $_{\Delta 117-118}$ regulated flagellar gene expression in a similar pattern as wild-type FliX did, albeit the overall activity of the reporter genes was lower, which could be due to the low cellular level of this mutant (Figure 4).

Effect of conserved FliX residues on cell morphology In Caulobacter, cells bearing mutations in early class II flagellar genes, including $f l i X$, exhibit a cell division phenotype consisting of filamentous growth at the onset of late log phase [38]. As shown in Figure 7, the culture of JG1172 was dominated by filamentous cells, whereas JG1172 cells expressing the wild-type fliX gene had normal cell morphology. All fliX mutants, except fliX $X_{L 85 K}$ (Figure 7), were able to restore the normal pattern of cell division in JG1172 cells. Once more, fli $X_{\mathrm{L} 85 \mathrm{~K}}$ displayed no activity in complementing a physiological defect in $\Delta f l i X$ cells.

Role of conserved FliX residues in interaction with FlbD Based on previous findings [35,37] and the new evidence of this study (Figure 1), it has been conclusively 


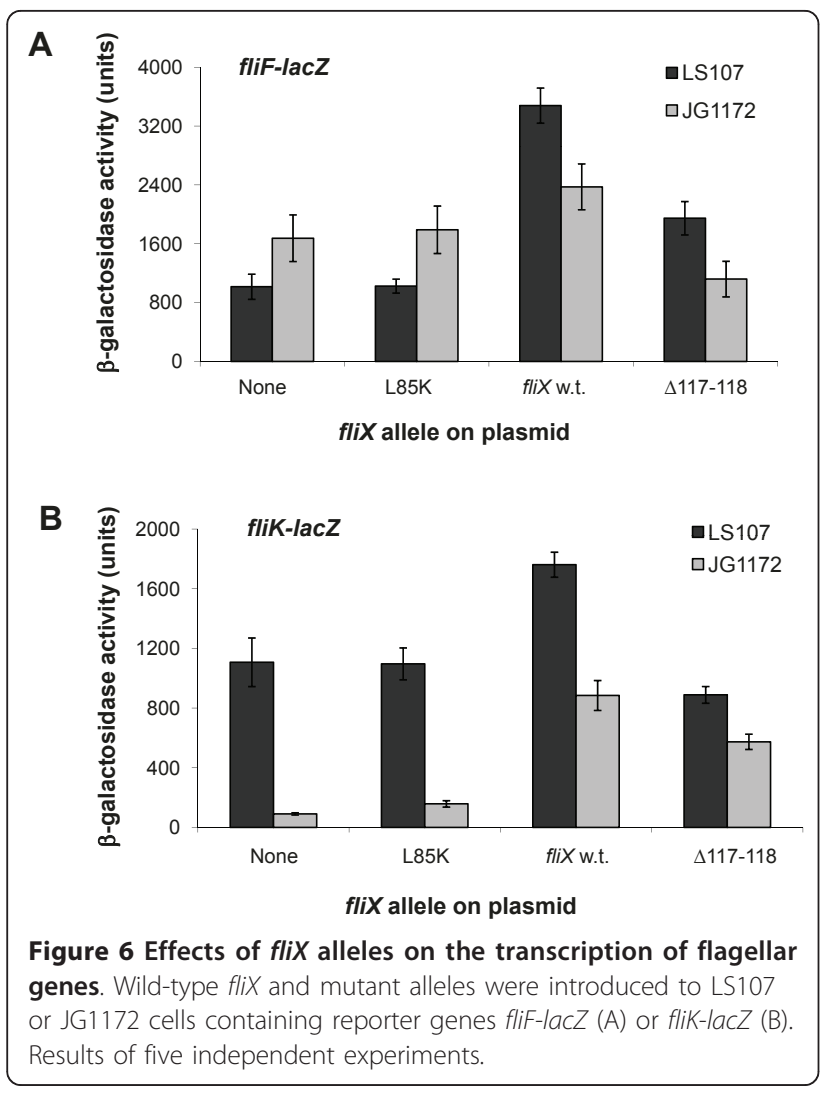

established that FliX and FlbD bind each other both in vivo and in vitro. The above genetic analyses revealed that although the cellular contents of FliX $\mathrm{X}_{\Delta 117-118}$ and FliX $_{\mathrm{L} 85 \mathrm{~K}}$ were similar in a $\Delta f l i X$ background, they exerted distinctive effects on FlbD activity. Their ability to interact with FlbD must have played a role. To test this idea, we performed an immunoprecipitation
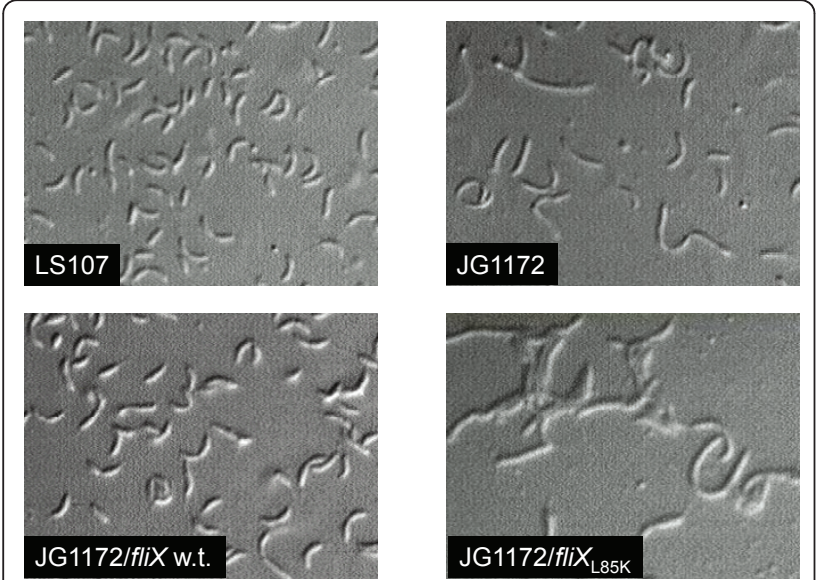

Figure 7 Allele fliX $\mathrm{L}_{\mathrm{L} 5 \mathrm{~K}}$ was unable to rescue the cell division defect of JG1172. Cells harvested from overnight cultures were mounted on poly-L-lysine coated slides and examined by differential interference contrast (DIC) microscopy. experiment in which cell extracts of Caulobacter were treated with agarose beads coated with anti-FlbD antibodies, and elutes from the beads were probed with antiFliX antibodies. As presented in Figure 8, mutants FliX $_{\mathrm{R} 71 \mathrm{~A}}, \mathrm{FliX}_{\mathrm{T} 130 \mathrm{~L}}$, and $\mathrm{FliX}_{\mathrm{L} 136 \mathrm{~K}}$ interacted as well with FlbD as wild-type FliX did, if their cellular contents (Figure 4) were taken into consideration. In spite of the fact that $\mathrm{FliX}_{\mathrm{L} 85 \mathrm{~K}}$, FliX $\mathrm{X}_{\triangle 117-118}$, and FliX 1 were maintained at similar protein levels in JG1172 cells (Figure 4, lanes 13,14 , and 17), the precipitated amounts of these proteins were dramatically different (Figure 8 , lanes 6,7 , and 10). Abundant FliX 1 and a small amount of FliX $_{\Delta 117-118}$ were obtained, whereas no detectable level of FliX $\mathrm{L}_{\mathrm{L} 8 \mathrm{~K}}$ was observed. This indicates that FliX 1 has a strong association to FlbD, FliX $\mathrm{X}_{\Delta 117-118}$ binds to FlbD with a low affinity, and FliX $\mathrm{L}_{\mathrm{L} 85 \mathrm{~K}}$ no longer interacts with FlbD. Since a large amount of FliX 1 was successfully precipitated, the results of this experiment reflect a lack of interaction between FlbD and FliX $_{\mathrm{L} 85 \mathrm{~K}}$ rather than a lack of FliX $\mathrm{L}_{\mathrm{LSK}}$ protein in the cell extracts.

\section{Discussion}

The interaction between FliX and FlbD, two class II flagellar regulatory proteins in Caulobacter, represents a novel mechanism for regulating the activity of a $\sigma^{54}$ transcription factor. The activities of many such factors are regulated by the phosphorylation of a conserved aspartate residue in their receiver domains $[42,43]$. However, the receiver domain of FlbD diverges substantially from others [37]. For example, it lacks some key residues necessary for the phosphorylation process [44]. No corresponding cognate histidine kinase for FlbD has been identified so far, and FlbD is active in the absence

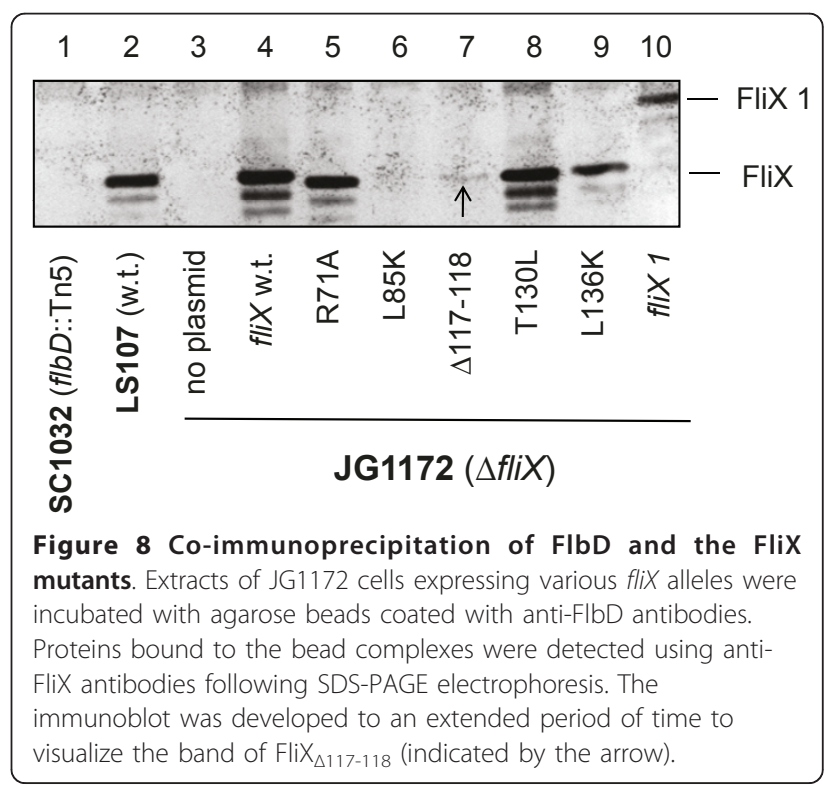


of phosphorylation [30,34]. In addition, purified FliX can regulate FlbD-activated transcription in vitro, probably by affecting the oligomerization state of FlbD [35]. In this study, we further demonstrated that through a remarkably high affinity, the two proteins bind to each other to perform their regulatory activity and to escape the fate of premature degradation. Mutations in conserved regions of FliX could interrupt the recognition between the two and hence their activity.

The observed low concentrations of FliX $\mathrm{L}_{\mathrm{L} 8 \mathrm{~K}}, \mathrm{FliX}_{\Delta 117 \text { - }}$ 118, and FliX 1 in JG1172 cells may be caused by their intrinsically low expression levels or their short half-life, or a combination of both. DNA or mRNA sequences of the alleles may carry intrinsic defects that inhibit the transcription or translation efficiency of the mutated genes. It is also possible that the mutations unfortunately expose target sites to intracellular proteases, making the gene products prone to degradation. Lack of protection from FlbD may also play a role in the case of FliX $_{\mathrm{L} 85 \mathrm{~K}}$. No matter what might be the main cause, the final result is that the cellular levels of the three are about the same (Figure 4). Nevertheless, their differential binding affinities to FlbD lead to dramatically different physiological outcomes. FliX $\mathrm{L}_{\mathrm{L} 85 \mathrm{~K}}$ completely losts the ability to interact with FlbD and exerts no influence to FlbD-mediated cellular processes. The fair amount of cellular $\mathrm{FliX}_{\mathrm{L} 85 \mathrm{~K}}$ (Figure 4) does not benefit the $\Delta$ fliX host in any observable way (Figure 5, 6 and 7). The mutation must have altered the gross structure of FliX and thus prevented an effective binding to FlbD. FliX $_{\Delta 117-118}$ can still interact with FlbD to a certain degree; therefore, it is largely functional in regulating FlbD activity (Figure 5 and 6). With a strong affinity to FlbD, FliX 1 becomes constitutively active; it turns on the transcription of class III/IV genes in the absence of the class II basal body $[37,38]$. The other three mutations, R71A, T130L, and L136K cause no significant effect to the expression of FliX, the interaction with FlbD, and hence the regulatory activity of the two partners.

Since the three dimensional structure of FliX (or a homolog) remains to be solved, it is still unclear which residues or regions of FliX and FlbD are in direct contact. An alanine scanning analysis should be helpful to probe the structural basis of the interaction. Relevant insights could also be gathered by selection for extragenic suppressors in FlbD that are specific to loss-offunction FliX alleles like FliX $\mathrm{L}_{\mathrm{L} 5 \mathrm{~K}}$.

\section{Conclusions}

Direct association of FliX and FlbD is required for their regulation on flagellar synthesis and other developmental events in Caulobacter. FliX and FlbD form high affinity complexes under physiological conditions, which is essential for the in vivo stability of each protein. Highly conserved regions of FliX are critical for binding to FlbD. Mutations in these regions could severely impact the recognition between the two and compromise their regulatory activity.

\section{Acknowledgements}

We are grateful to Dr. Jill Zeilstra-Ryalls at BGSU for helpful discussions. This work was supported by Public Health Service Grant GM48417 from the National Institutes of Health to JWG.

\section{Author details}

${ }^{1}$ Department of Chemistry and Biochemistry and Molecular Biology Institute, University of California, Los Angeles, CA 90095-1569, USA. ²Department of Biological Sciences and Center for Photochemical Sciences, Bowling Green State University, Bowling Green, OH 43403-0208, USA. ${ }^{3}$ FAS Center for Systems Biology, Harvard University, 52 Oxford Street, Cambridge, MA 02138, USA.

\section{Authors' contributions}

JWG conceived and coordinated the study and helped to draft the manuscript. RJD performed the protein stability assay. ZX carried out the rest experiments and drafted the manuscript. All authors participated in experiments designs and data analyses. All authors read and approved the final manuscript.

Received: 10 February 2011 Accepted: 2 May 2011

Published: 2 May 2011

\section{References}

1. Brun $\mathrm{W}$, Marczynski G, Shapiro L: The expression of asymmetry during Caulobacter cell differentiation. Annu Rev Biochem 1994, 63:419-450.

2. Gober JW, England J: Regulation of flagellum biosynthesis and motility in Caulobacter Prokaryotic Development.Edited by: Brun KV, Shimkets LJ. Washington, DC: American Society for Microbiology; 2000:319-339.

3. Gober JW, Marques MV: Regulation of cellular differentiation in Caulobacter crescentus. Microbiol Rev 1995, 59(1):31-47.

4. Wu J, Newton A: Regulation of the Caulobacter flagellar gene hierarchy; not just for motility. Mol Microbiol 1997, 24(2):233-239.

5. England JC, Gober JW: Cell cycle control of cell morphogenesis in Caulobacter. Curr Opin Microbiol 2001, 4(6):674-680.

6. Bryan R, Purucker M, Gomes SL, Alexander W, Shapiro L: Analysis of the pleiotropic regulation of flagellar and chemotaxis gene expression in Caulobacter crescentus by using plasmid complementation. Proc Nat Acad Sci USA 1984, 81(5):1341-1345.

7. Champer R, Dingwall A, Shapiro L: Cascade regulation of Caulobacter flagellar and chemotaxis genes. J Mol Biol 1987, 194(1):71-80.

8. Mangan EK, Bartamian M, Gober JW: A mutation that uncouples flagellum assembly from transcription alters the temporal pattern of flagellar gene expression in Caulobacter crescentus. J Bacteriol 1995, 177(11):3176-3184.

9. Minnich SA, Newton A: Promoter mapping and cell cycle regulation of flagellin gene transcription in Caulobacter crescentus. Proc Natl Acad Sci USA 1987, 84(5):1142-1146.

10. Newton A, Ohta N, Ramakrishnan G, Mullin D, Raymond G: Genetic switching in the flagellar gene hierarchy of Caulobacter requires negative as well as positive regulation of transcription. Proc Natl Acad Sci USA 1989, 86(17):6651-6655.

11. Ohta N, Chen LS, Mullin DA, Newton A: Timing of flagellar gene expression in the Caulobacter cell cycle is determined by a transcriptional cascade of positive regulatory genes. J Bacteriol 1991, 173(4):1514-1522.

12. Ohta $N$, Chen LS, Swanson $E$, Newton A: Transcriptional regulation of a periodically controlled flagellar gene operon in Caulobacter crescentus. J Mol Biol 1985, 186(1):107-115

13. Ramakrishnan G, Zhao JL, Newton A: Multiple structural proteins are required for both transcriptional activation and negative autoregulation of Caulobacter crescentus flagellar genes. J Bacteriol 1994, 176(24):7587-7600. 
14. $\mathrm{Xu} \mathrm{H}$, Dingwall $A$, Shapiro L: Negative transcriptional regulation in the Caulobacter flagellar hierarchy. Proc Natl Acad Sci USA 1989, 86(17):6656-6660.

15. Curtis PD, Brun YV: Getting in the loop: regulation of development in Caulobacter crescentus. Microbiol Mol Biol Rev 2010, 74(1):13-41.

16. Quon KC, Marczynski GT, Shapiro L: Cell cycle control by an essential bacterial two-component signal transduction protein. Cell 1996, 84(1):83-93

17. Reisenauer A, Quon K, Shapiro L: The CtrA response regulator mediates temporal control of gene expression during the Caulobacter cell cycle. J Bacteriol 1999, 181(8):2430-2439.

18. Domian IJ, Quon KC, Shapiro L: Cell type-specific phosphorylation and proteolysis of a transcriptional regulator controls the G1-to-S transition in a bacterial cell cycle. Cell 1997, 90(3):415-424

19. Anderson DK, Newton A: Posttranscriptional regulation of Caulobacter flagellin genes by a late flagellum assembly checkpoint. J Bacterio/ 1997 179(7):2281-2288.

20. Anderson PE, Gober JW: FlbT, the post-transcriptional regulator of flagellin synthesis in Caulobacter crescentus, interacts with the 5 untranslated region of flagellin mRNA. Mol Microbiol 2000, 38(1):41-52.

21. Mangan EK, Malakooti J, Caballero A, Anderson P, Ely B, Gober JW: FlbT couples flagellum assembly to gene expression in Caulobacter crescentus. J Bacteriol 1999, 181(19):6160-6170.

22. Llewellyn M, Dutton RJ, Easter J, O'Donnol D, Gober JW: The conserved flaF gene has a critical role in coupling flagellin translation and assembly in Caulobacter crescentus. Mol Microbiol 2005, 57(4):1127-1142.

23. Mullin D, Minnich S, Chen LS, Newton A: A set of positively regulated flagellar gene promoters in Caulobacter crescentus with sequence homology to the nif gene promoters of Klebsiella pneumoniae. Journal of molecular biology 1987, 195(4):939-943.

24. Gober JW, Shapiro L: Integration host factor is required for the activation of developmentally regulated genes in Caulobacter. Genes Dev 1990, 4(9):1494-1504.

25. Gober JW, Shapiro L: A developmentally regulated Caulobacter flagellar promoter is activated by $3^{\prime}$ enhancer and IHF binding elements. Mol Biol Cell 1992, 3(8):913-926.

26. Mullin DA, Newton A: Ntr-like promoters and upstream regulatory sequence $\mathrm{ftr}$ are required for transcription of a developmentally regulated Caulobacter crescentus flagellar gene. Journal of bacteriology 1989, 171(6):3218-3227

27. Mullin DA, Newton A: A sigma 54 promoter and downstream sequence elements ftr 2 and $\mathrm{ftr} 3$ are required for regulated expression of divergent transcription units flaN and flbG in Caulobacter crescentus. Journal of bacteriology 1993, 175(7):2067-2076.

28. Gober JW, Xu H, Dingwall AK, Shapiro L: Identification of cis and transelements involved in the timed control of a Caulobacter flagellar gene. Journal of molecular biology 1991, 217(2):247-257.

29. Benson AK, Ramakrishnan G, Ohta N, Feng J, Ninfa AJ, Newton A: The Caulobacter crescentus FlbD protein acts at ftr sequence elements both to activate and to repress transcription of cell cycle-regulated flagellar genes. Proc Natl Acad Sci USA 1994, 91(11):4989-4993.

30. Benson $\mathrm{AK}, \mathrm{Wu} \mathrm{J}$, Newton $\mathrm{A}$ : The role of FlbD in regulation of flagellar gene transcription in Caulobacter crescentus. Res Microbiol 1994, 145(56):420-430.

31. Mullin DA, Van Way SM, Blankenship CA, Mullin AH: FlbD has a DNAbinding activity near its carboxy terminus that recognizes $\mathrm{ftr}$ sequences involved in positive and negative regulation of flagellar gene transcription in Caulobacter crescentus. J Bacteriol 1994 176(19):5971-5981.

32. Ramakrishnan G, Newton A: FlbD of Caulobacter crescentus is a homologue of the NtrC (NRI) protein and activates sigma 54-dependent flagellar gene promoters. Proc Natl Acad Sci USA 1990, 87(6):2369-2373.

33. Wingrove JA, Mangan EK, Gober JW: Spatial and temporal phosphorylation of a transcriptional activator regulates pole-specific gene expression in Caulobacter. Genes Dev 1993, 7(10):1979-1992.

34. Wu J, Benson AK, Newton A: Global regulation of a sigma 54-dependent flagellar gene family in Caulobacter crescentus by the transcriptional activator FlbD. J Bacteriol 1995, 177(11):3241-3250.

35. Dutton RJ, Xu Z, Gober JW: Linking structural assembly to gene expression: a novel mechanism for regulating the activity of a sigma54 transcription factor. Mol Microbiol 2005, 58(3):743-757.
36. Muir RE, Gober JW: Mutations in FlbD that relieve the dependency on flagellum assembly alter the temporal and spatial pattern of developmental transcription in Caulobacter crescentus. Mol Microbiol 2002, 43(3):597-615.

37. Muir RE, Gober JW: Regulation of FlbD activity by flagellum assembly is accomplished through direct interaction with the trans-acting factor, FliX. Mol Microbiol 2004, 54(3):715-730.

38. Muir RE, O'Brien TM, Gober JW: The Caulobacter crescentus flagellar gene, fliX, encodes a novel trans-acting factor that couples flagellar assembly to transcription. Mol Microbiol 2001, 39(6):1623-1637.

39. Poindexter JS: Biological Properties and Classification of the Caulobacter Group. Bacteriol Rev 1964, 28:231-295

40. Miller JH: A short course in bacterial genetics: A Laboratory Manual and Handbook for Escherichia coli and Related Bacteria. Cold Spring Harbor Laboratory Press, Cold Spring Harbor, NY 1992.

41. Ohta N, Swanson E, Ely B, Newton A: Physical mapping and complementation analysis of transposon Tn5 mutations in Caulobacter crescentus: organization of transcriptional units in the hook gene cluster. J Bacteriol 1984, 158(3):897-904.

42. Gu B, Lee JH, Hoover TR, Scholl D, Nixon BT: Rhizobium meliloti DctD, a sigma 54-dependent transcriptional activator, may be negatively controlled by a subdomain in the C-terminal end of its two-component receiver module. Mol Microbiol 1994, 13(1):51-66.

43. Lee SY, De La Torre A, Yan D, Kustu S, Nixon BT, Wemmer DE: Regulation of the transcriptional activator $\mathrm{NtrC1}$ : structural studies of the regulatory and AAA+ ATPase domains. Genes Dev 2003, 17(20):2552-2563.

44. Volz K: Structural conservation in the CheY superfamily. Biochemistry 1993, 32(44):11741-11753.

45. Stephens C, Mohr C, Boyd C, Maddock J, Gober J, Shapiro L: Identification of the flil and fliJ components of the Caulobacter flagellar type III protein secretion system. J Bacterio/ 1997, 179(17):5355-5365.

46. Simon R, Priefer U, Puhler A: A Broad Host Range Mobilization System for In Vivo Genetic Engineering: Transposon Mutagenesis in Gram Negative Bacteria. Nat Biotech 1983, 1(9):784-791.

47. Kovach ME, Phillips RW, Elzer PH, Roop RM, Peterson KM: pBBR1MCS: a broad-host-range cloning vector. Biotechniques 1994, 16(5):800-802.

48. Wingrove JA, Gober JW: A sigma 54 transcriptional activator also functions as a pole-specific repressor in Caulobacter. Genes \& development 1994, 8(15):1839-1852

doi:10.1186/1471-2180-11-89

Cite this article as: Xu et al:: Direct interaction of FliX and FlbD is required for their regulatory activity in Caulobacter crescentus. BMC Microbiology 2011 11:89.

\section{Submit your next manuscript to BioMed Central and take full advantage of:}

- Convenient online submission

- Thorough peer review

- No space constraints or color figure charges

- Immediate publication on acceptance

- Inclusion in PubMed, CAS, Scopus and Google Scholar

- Research which is freely available for redistribution
C Biomed Central 